\title{
Low-frequency ultrasound-mediated microvessel disruption combined with docetaxel to treat prostate carcinoma xenografts in nude mice: A novel type of chemoembolization
}

\author{
YU YANG*, WENKUN BAI*, YINI CHEN, SHULIANG NAN, YANDUAN LIN, TAO YING and BING HU \\ Department of Ultrasound in Medicine, Sixth People's Hospital Affiliated to Shanghai Jiao Tong University, \\ Shanghai Institute of Ultrasound in Medicine, Shanghai 200233, P.R. China
}

Received March 31, 2015; Accepted May 6, 2016

DOI: $10.3892 / 01.2016 .4703$

\begin{abstract}
The aim of the present study was to investigate whether low-frequency ultrasound (US)-mediated microvessel disruption combined with docetaxel (DTX) can be used as a novel type of chemoembolization. Mice were assigned to four groups: i) The USMB group, treated with low-frequency US combined with microbubbles (USMB); ii) the DTX group, treated with DTX; iii) the USMB + DTX group, treated with combined therapy; and iv) the control group, which was untreated. Immediately after the first treatment, the average peak intensity (API) on contrast-enhanced US was calculated, and tumors were excised for hematoxylin and eosin (HE) staining. At 2 weeks post-treatment, the tumor volumes and wet weights were calculated, and tumors were excised for immunohistochemistry to calculate apoptotic index (AI), proliferative index (PI) and microvessel density (MVD) values. Immediately after the first treatment, in the DTX and control groups, the tumors demonstrated abundant perfusion enhancement, while in the USMB + DTX and USMB groups, blood perfusion of the tumors was interrupted. Compared with that of the control group, the API was significantly lower in the USMB + DTX USMB groups (all P<0.001). HE staining showed that tumor microvasculature was disrupted into flaky hematomas and severely dilated microvessels in the USMB + DTX and USMB groups. In the DTX and control groups, there was no distinct evidence of the disruption and
\end{abstract}

Correspondence to: Professor Bing Hu and Professor Tao Ying, Department of Ultrasound in Medicine, Sixth People's Hospital Affiliated to Shanghai Jiao Tong University, Shanghai Institute of Ultrasound in Medicine, 600 Yi Shan Road, Shanghai 200233, P.R. China

E-mail: hubingedu@hotmail.com

E-mail: yingtaotao666@126.com

*Contributed equally

Key words: low-frequency ultrasound, docetaxel, microbubble, prostate carcinoma, tumor vasculature dilation of blood microvessels. At the end of the treatment, the mean tumor inhibition ratio was $73.33,46.67$ and $33.33 \%$ for the USMB + DTX, DTX and USMB groups, respectively. The USMB + DTX group had the highest AI, and the lowest PI and MVD compared with the other groups, although the difference between the USMB + DTX and DTX groups with regard to PI and MVD was not significant (USMB + DTX vs. DTX group, $\mathrm{P}=0.345$ and $\mathrm{P}=0.059$, respectively). In conclusion, as a novel type of chemoembolization, USMB combined with DTX is more effective than USMB or DTX alone in inhibiting tumor growth via the enhancement of apoptosis, and the suppression of proliferation and angiogenesis.

\section{Introduction}

The morbidity of prostate cancer is estimated as the highest among all American male malignancies. The disease is the second highest cause of tumor-associated mortality among American men, accounting for $27 \%$ (233,000 cases) of all newly diagnosed cancer cases in men and 10\% (29,480 cases) of all male cancer-associated mortalities in 2014 in American (1). Due to a lack of early symptoms, numerous cases are diagnosed in the advanced stages. During the advanced stages, the majority of patients receive hormone therapy, such as androgen-receptor antagonist treatment (2). Responses to hormone therapy are initially encouraging. However, a large percent of androgen-dependent cancers will gradually become resistant to castration, i.e., castration-resistant prostate cancer (CRPC) (2). At this stage, second-line endocrine therapy and chemotherapy should be administered (3).

Docetaxel (DTX) is a clinically approved drug for the treatment of various metastatic CRPCs (4). The majority of metastatic prostate cancers can be effectively treated with DTX (5). DTX promotes microtubule polymerization, which can cause the cell cycle to arrest at the $\mathrm{G}_{2} / \mathrm{M}$ phase and ultimately leads to apoptosis or death in cancer cells (6). In addition, DTX has been reported to exert antiangiogenic effects (7). However, the systemic administration of DTX exposes all tissues to cytotoxic drugs (3). This side-effects of systemic chemotherapy highlights the requirement for improved and targeted delivery to aid in increased localized drug uptake in the targeted cancer cells. Although this problem could be 
solved by transarterial arterial chemoembolization (TACE), this therapy also has certain deficiencies; for example, it is difficult to completely embolize the tumor-feeding vessels due to the complexity of the blood supply and the limitations of the TACE technique.

As a highly effective, low-cost and non-invasive tool, low-frequency ultrasound combined with microbubbles (USMB) has the capacity of inducing drugs to accumulate in malignant tissues and reducing the toxic effects upon systemic administration to healthy tissues (3). Unlike in previous studies on USMB in combination with antitumor agents, which have largely focused on promoting uptake though enhancing permeability $(3,8)$, the present study used USMB as an embolic 'agent' to retain chemotherapeutic drugs, in a similar manner to TACE. In theory, the mechanisms of chemoembolization using USMB include two aspects. Firstly, due to the physiologically defects in tumor neovasculature (9), USMB can selectively shut down the blood flow of tumors without having an adverse impact on normal tissues (10-12), and this is similar to embolic agents in TACE. Secondly, extensive and severe dilated microvessels could entrap a large volume of chemotherapeutic drugs, which play the role of drugs in TACE. We suspect that this technology may be promising to treat CRPC.

The present study analyzed the combination of USMB treatment and DTX, aiming to develop a safe and effective type of chemoembolization. Experiments were conducted on prostate carcinoma PC3 xenografts in nude mice. The acute anti-vascular effects of treatments on tumor perfusion and tumor vasculature destruction were investigated. At the end of this experiment, tumor growth was compared and immunohistochemistry was performed to evaluate cell apoptosis, cell proliferation and angiogenesis in the prostate carcinoma xenografts.

\section{Subjects and methods}

Cell lines and xenograft tumor model. The present study was approved by the Institutional Review Board of the Sixth People's Hospital (Affiliated to Shanghai Jiao Tong University, Shanghai Institute of Ultrasound in Medicine, Shanghai, China). The PC3 cell line, consisting of human androgen-independent prostate cancer cells, was obtained from the Cell Bank of the Chinese Academy of Sciences (Shanghai, China). The cells were cultured in Dulbecco's modified Eagle's medium (HyClone, Logan, UT, USA), which was added to $10 \%$ fetal bovine serum in an incubator with $5 \% \mathrm{CO}_{2}$ at $37^{\circ} \mathrm{C}$. When the cells reached $80 \%$ confluence, they were washed with phosphate-buffered saline (PBS), trypsinized (trypsin; Ginuo Biomedical Technology Co. Ltd., Hangzhou, China) and centrifuged at $150 \mathrm{x} \mathrm{g}$ at $25^{\circ} \mathrm{C}$ for $5 \mathrm{~min}$. Thereafter, the cells were resuspended in PBS, and the final viable cell solution was estimated at a concentration of $1 \times 10^{7}$ cells per $100 \mu \mathrm{l}$.

A total of 60 male Balb-c nude mice (age, 5-6 weeks old; weight, 20-25 g) were purchased from Shanghai Super-B\&K Laboratory Animal Corporation, Ltd., (Shanghai, China) and raised in the Animal Laboratory of Shanghai Jiao Tong University Affiliated Sixth People's Hospital. The mice were fed with sufficient chow and water for a week prior to being injected with the prostate cancer PC3 cells. Each treatment, including establishing the animal model and the therapeutic treatment, was performed under general anesthesia by intraperitoneal injection of $50 \mathrm{mg} / \mathrm{kg}$ of $1.5 \%$ pentobarbital sodium and local sterilization. Each mouse was subcutaneously inoculated with $1 \times 10^{7}$ cells from the PC 3 cell line into the right flank. The mice continued to be raised under specified pathogen-free conditions subsequent to the procedure, and were observed at 2-day intervals. Experiments were initiated 2 weeks later, when the tumors had grown to 4-6 $\mathrm{mm}$ in maximum diameter in 52 of the nude mice, and these 52 mice were subsequently recruited in the experiment. There was no significant difference in tumor volumes across all groups at the beginning of treatment.

Treatment protocols. Animals were randomly divided into 4 groups of 13 animals each: The USMB + DTX group was treated with combined therapy (DTX followed by USMB), the USMB group was treated with USMB only, the DTX group was treated with DTX only and the control group was not treated. For the USMB + DTX group, DTX was injected $10 \mathrm{~min}$ prior to USMB treatment (12). Immediately after the first treatment, 5 mice were randomly selected from each group to undergo contrast-enhanced US (CEUS) and hematoxylin and eosin (HE; Beijing Leagene Biotech. Co., Ltd., Beijing, China) staining to elucidate mechanisms. The remaining animals were treated 4 times a day at a treatment interval of $3 \mathrm{~h}$. Treatments were applied every other day for 1 week. Furthermore, 2 weeks after the first treatment, the mice underwent two-dimensional (2D) US to evaluate the tumor volumes, and then the tumors were surgically excised to analyze the pathological changes. At the end of the experiment, all mice were euthanized by an overdose injection of sodium pentobarbital (150 mg/kg; Merck Millipore, Darmstadt, Germany).

DTX. DTX (Sima Lab Science Co., Ltd., Tianjin, China) was resolved in normal sodium to the concentration of $2 \mathrm{mg} / \mathrm{ml}$ and was administered at a dose of $10 \mathrm{mg} / \mathrm{kg}$ as a single bolus injection through the tail vein. As USMB can be utilized as a chemoembolization method to entrap drugs only if there are chemotherapeutic agents in circulation, in this regard it is vital that the DTX injection occurred $10 \mathrm{~min}$ prior to USMB (13).

Treatment with USMB. Microbubbles contain gas encased in a shell, and they are capable of passing through the capillary lumen. Albumin-coated microbubbles, termed perfluoropropane-albumin microsphere injections (Runken Pharmaceutical Co., Yueyang, China), were used for CEUS and therapeutic application. The microbubbles had a mean diameter of $3.4 \mu \mathrm{m}$, with $99 \%$ of the particles $<10 \mu \mathrm{m}$ in diameter and a microbubble concentration of $6.5 \times 10^{8} / \mathrm{ml}$. Agitation was gently applied for $\sim 20 \mathrm{sec}$ prior to the milky white suspension being ready. For CEUS, a bolus injection of $0.10 \mathrm{ml}$ microbubbles was injected into each mouse through the tail vein. For the vessel blocking treatment, a bolus injection of $0.20 \mathrm{ml}$ microbubbles was injected into each mouse through the tail vein.

The low-frequency US equipment was manufactured by the Shanghai Institute of Ultrasound in Medicine. The 
diameter of the therapeutic US transducer was $20 \mathrm{~mm}$, which covered the entire tumor. The therapeutic parameters were determined by our previous orthogonal experimental design as follows: Frequency, $20 \mathrm{kHz}$; acoustic intensity, $1 \mathrm{~W} / \mathrm{cm}^{2}$; duty cycle, $40 \%$ ( $2 \mathrm{sec}$ on, $3 \mathrm{sec}$ off); and irradiation time, 3 min (14). The intermittent working mode allowed microbubbles to refill after every microbubble was destroyed. The transducer was positioned above the tumors, but did not press upon them. The space between the tumor and the transducer was filled with coupling gel. Following the intravenous injection of the microbubbles $(0.20 \mathrm{ml})$, the tumors were insonated percutaneously with low-frequency US for $3 \mathrm{~min}$.

Average peak intensity (API) by CEUS. A commercially available US imaging system, Mylab90 instrument (Esaote, Genoa, Italy) equipped with LA522 and LA523 high frequency linear array probes, was used to calculate the API by CEUS immediately after first treatment and to measure the tumor diameters on the $2 \mathrm{D}$ images at the end of the treatment, respectively. For CEUS, dual-frame imaging combining 2D and contrast modality was displayed using a low mechanical index (0.05). Depth, frequency and other US conditions were kept the same during all CEUS studies. The section was the largest available section of each tumor determined according to 2D images; this enabled the selected section to reflect the blood perfusion changes of the whole tumor, including central and peripheral regions. The real-time CEUS dynamic images of these planes were recorded for $3 \mathrm{~min}$ at a time following an intravenous bolus injection of microbubbles. The dynamic images were stored in the built-in hard disk of the machine, then exported for contrast analysis.

The CEUS images were analyzed by the QontraXt Software (Esaote, Genoa, Italy) to determine the API. The QontraXt Software quantification interface displays the 2D and CEUS images. The boundary of the tumor was drawn by clicking the left mouse button on the CEUS images of the first frame for the region of interest (ROI), and no great breathing movement was noted during CEUS. The first three min was defined, then a time-intensity curve was automatically generated. The API was calculated automatically. The API represents the average echogenicity within an ROI at the peak contrast enhancement.

Tumor size calculation. The recorded diameters of the tumors on the 2D US images were based on the consensus of two observers. Tumor volumes were calculated using the following formula: Tumor volume $\left(\mathrm{mm}^{3}\right)=\left(\mathrm{a} \mathrm{x} \mathrm{b}^{2}\right) / 2$, where $a$ and $b$ are the longest and shortest diameters of the measured tumor, respectively. Next, the mice were anesthetized, and the tumors were harvested and weighted. The tumor inhibition ratio was calculated using the formula: Tumor inhibition ratio $(\%)=(\mathrm{w} 1-\mathrm{w} 2) / \mathrm{w} 1$, where $\mathrm{w} 1$ and $\mathrm{w} 2$ are the mean tumor wet weights of the control and treatment groups, respectively.

Sample collection and pathological examination. The harvested tumor specimens were fixed in $10 \%$ neutral formalin (Nanchang Yulu Experimental Equipment Co., Ltd., Nanchang, China) for $24 \mathrm{~h}$, then the tissues were embedded in paraffin and $5-\mu \mathrm{m}$ thick sections were obtained for $\mathrm{HE}$ staining, tumor apoptosis analysis, detection of cell proliferation and angiogenesis. A pathologist blinded to the study evaluated tissue morphology changes with a light microscope (CX41; Olympus, Tokyo, Japan).

Apoptosis of the tumor cells was determined by terminal deoxynucleotidyl transferase-mediated deoxyuridine triphosphate nick-end labeling (TUNEL), using a commercially available kit (In Situ Cell Death Detection kit, POD; Roche Diagnostics GmbH, Mannheim, Germany). The total number of tumor cell nuclei and TUNEL-positive cell nuclei was counted at x400 magnification. TUNEL-positive nuclei were stained brown or tan. At least five, random, non-overlapping fields per tumor were analyzed, with a total of at least 500 nuclei for TUNEL staining. The apoptotic index (AI) was defined as the ratio of TUNEL-positive tumor cell nuclei to all tumor cell nuclei, and was expressed as a percentage.

Immunohistochemical quantification of tumor cell proliferation was determined using the anti-human Ki-67 rabbit monoclonal antibody (Abcam, Cambridge, UK; 1:500 dilution; catalog no. ab92742). Antigen retrieval was performed by the samples boiling in $10 \mathrm{mmol} / \mathrm{l}$ sodium citrate buffer for $15 \mathrm{~min}$. Non-specific binding sites were blocked with goat serum (Wuhan Boster Biological Engineering Co., Ltd., Wuhan, China) for $30 \mathrm{~min}$. The rabbit anti-Ki-67 antibody was applied overnight at $4^{\circ} \mathrm{C}$. Next, the slides were incubated with the goat anti-rabbit antibody (Zhongshan Golden Bridge Biotechnology, Beijing, China; 1:500 dilutions, catalog no. ZB-2301) for $30 \mathrm{~min}$ at $37^{\circ} \mathrm{C}$. The primary antibody was replaced with PBS as a negative control. The total number of tumor cell nuclei and Ki-67-positive cell nuclei was counted at x400 magnification. Ki-67-positive cell nuclei were stained brown or tan. Five, random, non-overlapping fields were analyzed with at least 500 nuclei counted from each section for Ki-67 staining. The proliferation index (PI) was defined as the ratio of $\mathrm{Ki}-67$-positive tumor cell nuclei to all tumor cell nuclei, and was expressed as a percentage.

Quantification of angiogenesis in the tumor tissues was determined by anti-mouse cluster of differentiation (CD)34 rabbit monoclonal antibody (Abcam; 1:250 dilution; catalog no. ab81289). Antigen retrieval was performed by boiling in $10 \mathrm{mmol} / \mathrm{l}$ sodium citrate buffer for $15 \mathrm{~min}$. Non-specific binding sites were blocked with goat serum (Wuhan Boster Biological Engineering Co., Ltd.) for $30 \mathrm{~min}$. The rabbit antiCD34 antibody was applied overnight at $4^{\circ} \mathrm{C}$. Next, the slides were incubated with a goat anti-rabbit antibody (Zhongshan Golden Bridge Biotechnology; 1:1,000 dilution; catalog no. ZB-2301) for $30 \mathrm{~min}$ at $37^{\circ} \mathrm{C}$. The primary antibody was replaced with $\mathrm{PBS}$ as a negative control. CD34 staining for blood vessels were observed under x400 magnification. For each slide, the microvessel density (MVD) was calculated as the number of CD34-positive vessels in five, random, non-overlapping fields.

Statistical analysis. All data are expressed as the mean \pm standard deviation. SPSS 19.0 (SPSS Inc., Chicago, IL, USA) was used to analyze all data. A one-way analysis of variance was used for comparisons of differences in API, tumor volume, AI, PI and MVD between the four groups. The least 


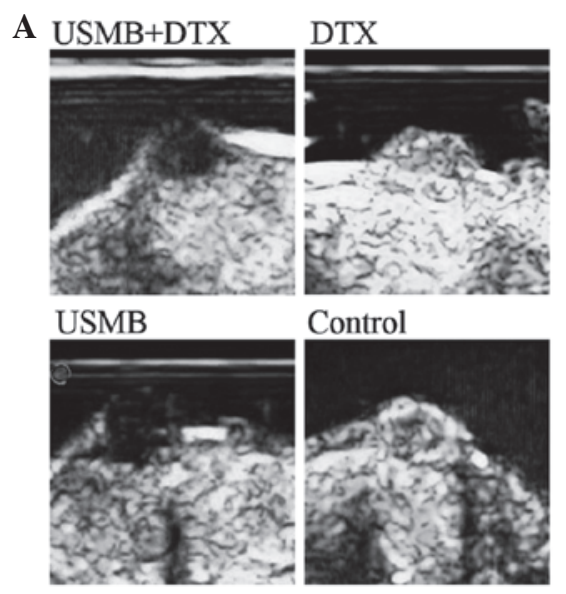

$\mathbf{B}$

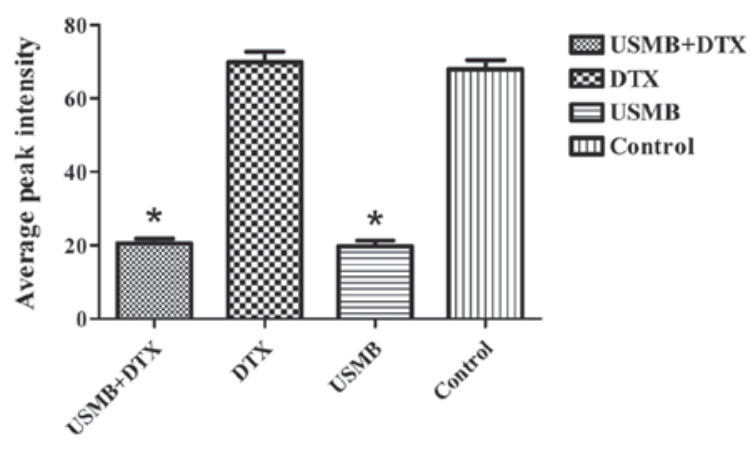

Figure 1. (A) Contrast-enhanced ultrasound imaging and (B) average peak intensity (API) of prostate cancer xenografts in nude mice in the USMB + DTX, USMB, DTX and control groups immediately after the first treatment. "P $<0.05$ vs. control group. Data are represented as the mean \pm standard deviation. USMB, low-frequency ultrasound combined with microbubbles; DTX, docetaxel.
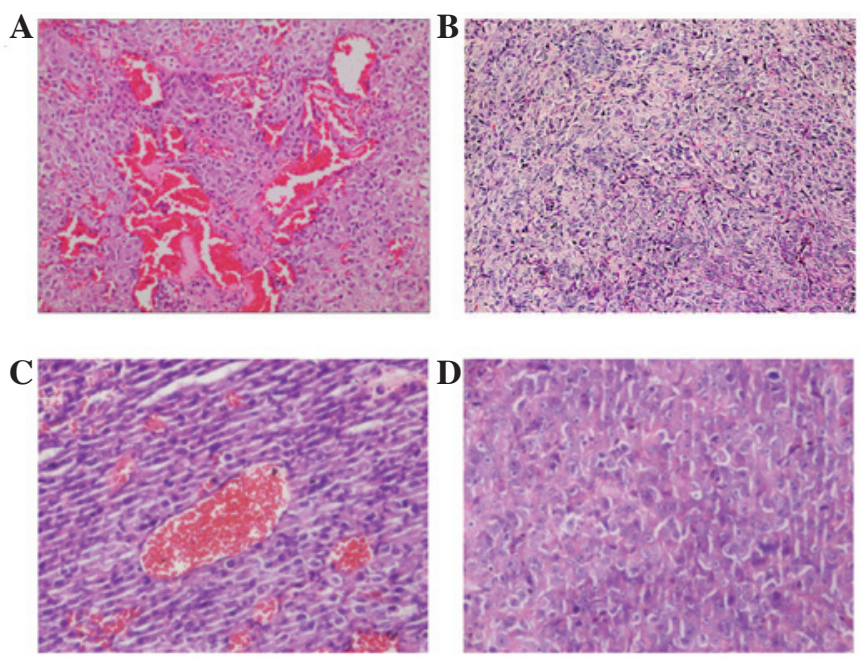

Figure 2. Hematoxylin and eosin staining of prostate cancer xenografts in nude mice in the USMB + DTX, USMB, DTX and control groups immediately after the first treatment. (A) Blood vessels were severely dilated and thrombi formed in the USMB + DTX group (magnification, $\mathrm{x} 200$ ). (B) Blood vessels were not evident in the DTX group (magnification, $\mathrm{x} 200$ ) (C) Microvessels were dilated to different degrees in the USMB group (magnification, $\mathrm{x} 400$ ). (D) Blood vessels were not evident in the control group (magnification, $x 400$ ). USMB, low-frequency ultrasound combined with microbubbles; DTX, docetaxel.

significant difference test was used for multiple comparisons. $\mathrm{P}<0.05$ was considered to indicate a statistically significant difference.

\section{Results}

Overview. All animals, except 1 mouse from the control group, survived the procedures until the designated time point of euthanasia. The efficacy of combining UMSB with DTX in the treatment of subcutaneous PC3 prostate cancer xenografts was evaluated immediately after the first treatment and at the end of all treatments, respectively.

API analysis. The implanted tumors were observed to be nodular in shape (Fig. 1A). Immediately after the first treatment, in the DTX and control groups, the tumors demonstrated abundant perfusion enhancement of the tumor vasculature. In the USMB + DTX and USMB groups, the tumor contrast perfusion was severely interrupted. The API in the USMB + DTX, DTX, USMB and control groups were calculated as $20.56 \pm 2.80,69.90 \pm 6.26,19.76 \pm 3.49$ and $67.96 \pm 5.52$, respectively (Fig. 1B). Compared with the control group, the API in the USMB + DTX and USMB groups was significantly lower (both $\mathrm{P}<0.001$ ). The difference between the USMB + DTX and USMB groups was not significant $(\mathrm{P}=0.793)$, and the difference between the DTX and control groups was not significant $(\mathrm{P}=0.526)$.

$H E$ staining. Immediately after the first treatment, on the HE-stained slides (Fig. 2), the PC3 prostate tumor in the DTX and control groups were apparent as invasive cancer nests; the tumors demonstrated a typical histological pattern characterized by loosely spaced and cord-like neoplastic cells forming a mass with reduced connective tissues and a sharply demarcated encapsulated border (Fig. 2B and D). Tumor necrosis was rare and not markedly evident. With the exception of occasional microvessels with a few red blood cells in the lumen, the blood vessels were almost inevident. In the USMB + DTX and USMB groups, the tumor microvasculature showed severe microvessel dilation (Fig. 2A and C), which could be accompanied by intravascular thrombosis. Vascular wall construction of certain tumor tissues was disrupted, resulting in a near disappearance of the blood wall structure. In addition, thrombosis and hematoma formed outside the vessel (Fig. 2A). Edema was frequently observed in the vicinity of the ruptured vessels, and the tumor cells were widely separated by edema. Furthermore, multiple pseudocysts in irregular round or oval appearances of various sizes were frequently observed in certain tumors. The cysts were delineated without endothelial lining around the border. Despite the vascular changes, the majority of the individual neoplastic cells appeared normal and alive.

Tumor volume calculation. To evaluate the effect of USMB and DTX on PC3 prostate cancer growth, the tumor volumes 
A

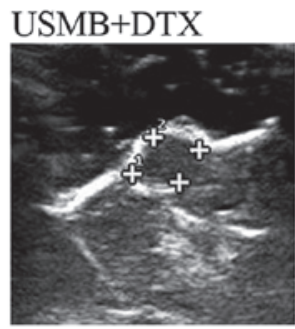

USMB

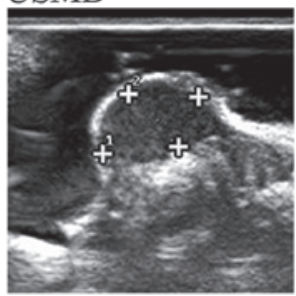

DTX

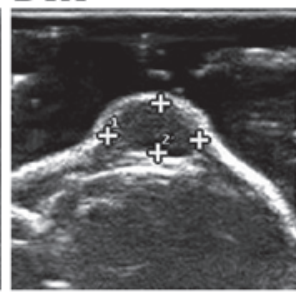

Control

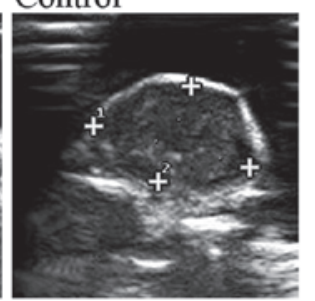

B

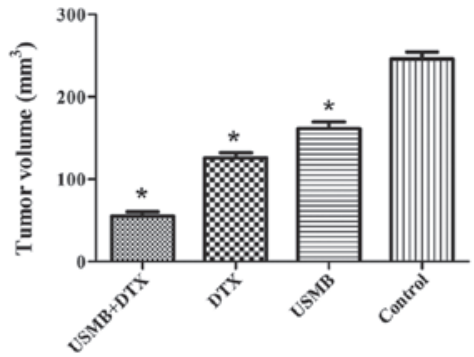

C

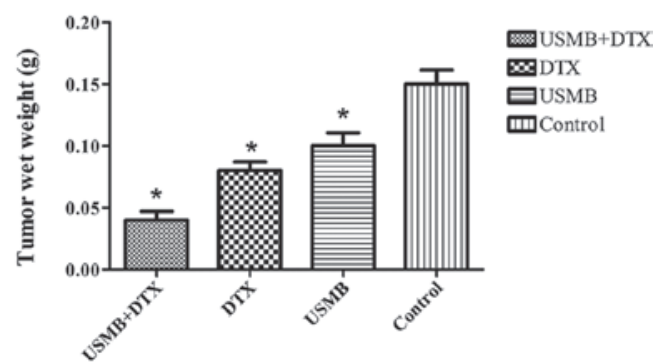

WMSB+DTX

$\varpi D T X$

曰USMB

血 Control

Figure 3. (A) Two-dimensional (2D) ultrasound images, (B) tumor volumes and (C) wet weights of xenografts in nude mice of the USMB + DTX, DTX, USMB and control groups at 2 weeks post-treatment. Data are represented as the mean \pm standard deviation. ${ }^{*} \mathrm{P}<0.05 \mathrm{vs}$. control group. USMB, low-frequency ultrasound combined with microbubbles; DTX, docetaxel.

were calculated for 2 weeks after the initial treatment using 2D ultrasonography. The implanted tumors were observed to be spherical- or elliptical in shape, and were well-defined, homogenous and hypoechoic on US images (Fig. 3A). The tumor volumes in the days after treatment are shown in Fig. 3B. In the PC3 xenografts, the mean absolute tumor volume at the end of treatment in the USMB + DTX, DTX, USMB and control groups was calculated as 55.01 \pm 14.93 , $125.88 \pm 17.78,161.36 \pm 22.40$ and $245.79 \pm 22.83 \mathrm{~mm}^{3}$, respectively. The combination of USMB and DTX produced the greatest tumor inhibition (compared with the other groups, all $\mathrm{P}<0.001)$. Tumor volumes were reduced in the USMB group when compared with the control group $(\mathrm{P}<0.001)$, while the DTX group produced even greater tumor volume inhibition (DTX vs. USMB group, $\mathrm{P}=0.001$ ).

Additionally, DTX treatment reduced the tumor wet weight compared with that of the control $(\mathrm{P}<0.001)$. However, there was no significant difference between the DTX and USMB groups $(\mathrm{P}=0.169)$ in terms of tumor wet weight. The combination of USMB and DTX produced a greater suppression of tumor wet weight than DTX and USMB alone ( $\mathrm{P}=0.005$ and $\mathrm{P}<0.001$, respectively) (Fig. $3 \mathrm{C}$ ). The mean tumor inhibition ratio of the USMB + DTX group was $73.33 \%$, which was significantly greater than that of the DTX (46.67\%) and USMB (33.33\%) groups. These results indicated that treatment with the combination of USMB and DTX is more effective than USMB or DTX alone in inhibiting tumor growth.

Tumor apoptosis. TUNEL assays of the subcutaneous tumors were performed to confirm the apoptotic effects induced by the different treatments (Fig. 4A). In the PC3 xenografts, the $\mathrm{AI}$ at the end of treatment in the USMB + DTX, DTX, USMB and control groups was calculated as $10.12 \pm 2.62,6.08 \pm 1.45$, $3.48 \pm 1.17$ and $2.25 \pm 1.14$, respectively (Fig. 4B). The AI of the USMB + DTX group was significantly higher than that of the other groups (all $\mathrm{P}<0.001$ ). The DTX group was also associated with a significant apoptotic effect compared with the controls $(\mathrm{P}<0.001)$, and the efficacy of DTX on the apoptotic effect was significantly more than that of USMB $(\mathrm{P}=0.005)$. Treatment with USMB alone conferred a higher AI compared with the control group, but the difference was not significant $(\mathrm{P}=0.182)$.

Immunohistochemical detection of cell proliferation. To determine the effects of treatment on tumor proliferation, immunohistochemical staining of Ki-67 was performed to quantify the PI in the tumors (Fig. 4A). The PI of the USMB + DTX, DTX, USMB and control groups was calculated as $1.72 \pm 1.67,3.83 \pm 1.82,21.71 \pm 5.75$ and $38.97 \pm 6.35$, respectively (Fig. 4C). The USMB + DTX group had the lowest PI compared with the other groups, although the difference between the USMB + DTX and DTX groups was not significant (USMB + DTX vs. DTX, P=0.345). The USMB group had a lower PI compared with the control group $(\mathrm{P}<0.001)$, but the efficacy of DTX on the apoptotic effect was significantly more than that of the USMB group $(\mathrm{P}<0.001)$.

Immunohistochemical detection of tumor angiogenesis. To determine the effects of treatment on tumor angiogenesis, immunohistochemical staining of CD34 was performed to quantify MVD in the tumors (Fig. 4A). The MVD of the USMB + DTX, DTX, USMB and control groups was calculated as $13.50 \pm 4.34,22.63 \pm 7.80,32.00 \pm 7.78$ and $47.71 \pm 14.87$, respectively (Fig. 4D). The USMB + DTX group had the lowest MVD compared with the other groups, although the difference between the USMB + DTX and DTX groups was not significant (USMB + DTX vs. DTX, P=0.059). The MVD of the USMB group was lower than that of the control group $(\mathrm{P}=0.003)$ and higher than that of the DTX group, but the difference was not significant between the USMB and DTX groups $(\mathrm{P}=0.053)$. 
A

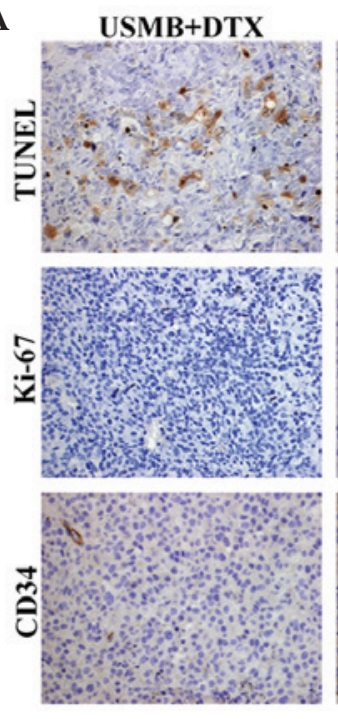

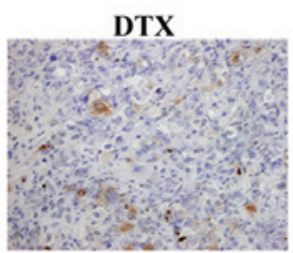
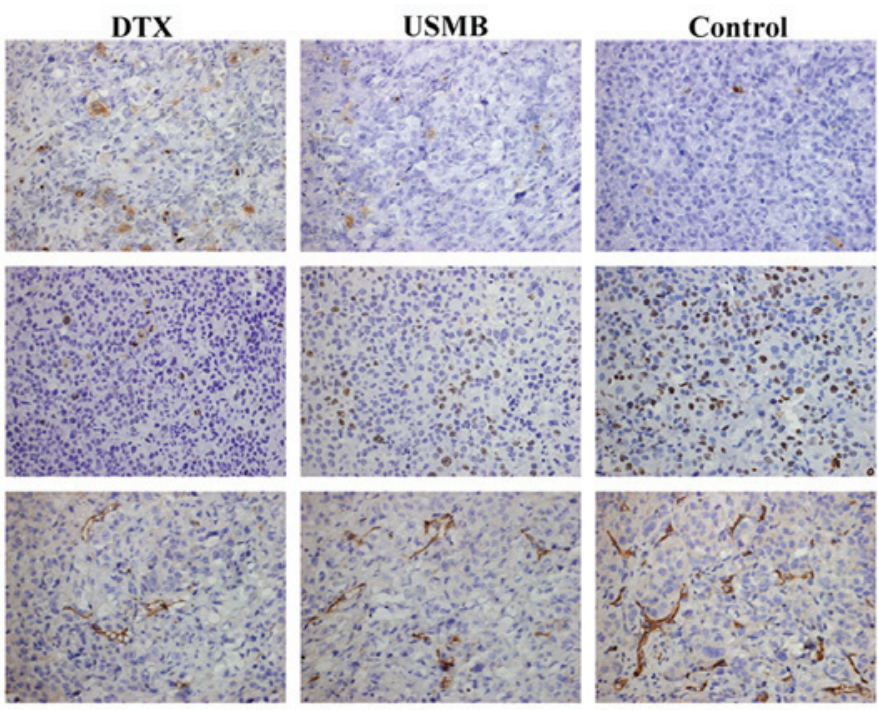

B

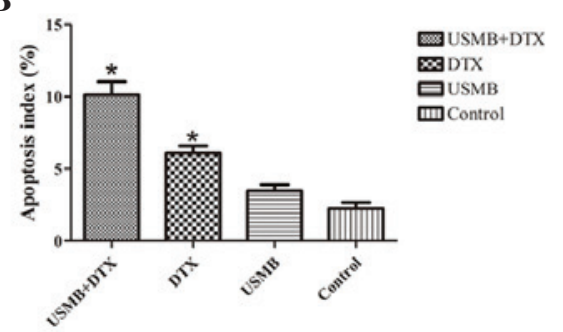

C

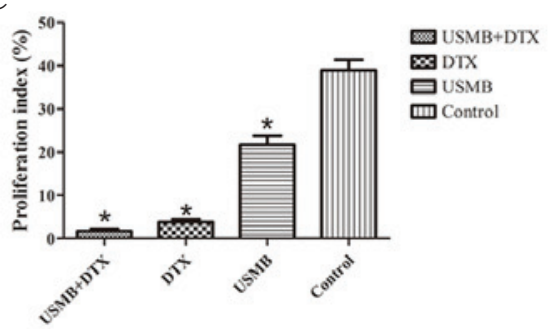

D

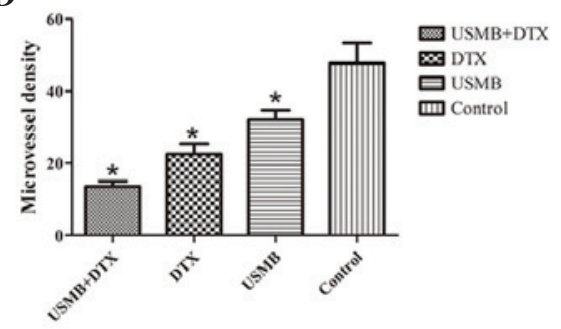

Figure 4. Cell apoptosis, cell proliferation and angiogenesis of prostate cancer tumor tissues of nude mice in the USMB + DTX, USMB, DTX and control groups at 2 weeks post-treatment. (A) Representative images of TUNEL and immunohistochemical staining of Ki-67 and CD34 of prostate cancer tumor tissues in four groups (magnification $\mathrm{x} 400$ ). (B) Apoptotic index, (C) proliferation index and (D) microvessel density in the four groups. * $\mathrm{P}<0.05$ vs. control group. Data are represented as the mean \pm standard deviation. USMB, low-frequency ultrasound combined with microbubbles; DTX, docetaxel; CD34, cluster of differentiation 34; TUNEL, terminal deoxynucleotidyl transferase-mediated deoxyuridine triphosphate nick-end labeling.

\section{Discussion}

Different studies have shown that microbubbles could induce significant bioeffects on confining vasculature (15-17). The bioeffects range from increasing the vascular permeability for the chemotherapy drug and target gene delivery by opening the blood-brain barrier locally and transiently, to vessel rupture and occlusion $(11,12,18-23)$. The antivascular effects of USMB can induce the interruption of blood flow in tumors and cause microvessels to dilate extensively. If a chemotherapeutic agent is circulating at the time of USMB treatment, the dilated vascular induced by USMB may entrap drugs within the tumor tissues. The present study investigated USMB as an embolic 'agent' in combination with DTX to achieve chemoembolization. We hypothesized that USMB may act as an embolic 'agent' and potentiate the antitumor effect of chemotherapy drugs. Conceptually, this is a strategy similar to TACE.

It has been demonstrated that thermal, mechanical (acoustic radiation forces, stable cavitation, inertial cavitation and other non-linear mechanisms) and sonochemical effects are likely to contribute to antivascular activities (9). In all these mechanisms, inertial cavitation and its corresponding effects are believed to be the dominating mechanism for microvessel damage. Transient cavitation and the resultant shock wave and fluid jets altogether bring about microvessel distention, invagination and ultimately vessel rapture $(15,17,24-26)$.
In the present study, immediately after the first treatment, CEUS in the USMB + DTX and USMB groups demonstrated that tumor blood flow blocking had occurred in response to USMB, which was consistent with the results of other studies using USMB to achieve blood perfusion interruption effects in tumors (10-12). HE staining showed that the tumor microvasculature was disrupted into flaky hematomas and severely dilated microvessels immediately after USMB treatment, whether in combination with DTX or not. The extensive and severe dilated microvessels could retain a large volume of chemotherapeutic drugs. In the DTX and control groups, there was no distinct evidence of the disruption or dilation of the blood microvessels.

USMB can improve vascular and cellular permeability, and increase the efficiency of chemotherapy through passive, localized delivery (3). The increased permeability of blood vessels and cell walls for the improved delivery of chemotherapy drugs can be achieved by microstreaming and radiation force, but in particular, by sonoporation $(23,27,28)$. Sonoporation, resulting from the violent collapse of microbubbles due to inertial cavitation, could generate fluid jet streams that can generate pores in the vessel walls and individual cells $(3,28)$, which can greatly facilitate the extravasation of drugs. Besides pore formation (29), other drug uptake routes could also be stimulated by USMB, including endocytosis (30) and the opening of cell-cell junctions $(31,32)$. Moreover, temperature 
elevation from absorption of US energy can lead to accelerated drug extravasation and intra-tissue diffusion (19). This technique of using USMB to transiently enhance membrane permeability could lead to improved tumor cell drug internalization. Furthermore, USMB can cause the capillary wall to burst, and in this process, ruptured vessels introduce microbubbles into the intravascular space (33), making it easier to induce ultrasonic cavitation in the extracellular matrix, thus enhancing drug delivery further. As expected, in the present study, USMB combined with DTX showed greater growth inhibition when compared with USMB or DTX alone. Semi-quantitative analysis of immunohistochemical staining showed that mice treated with USMB + DTX demonstrated increased cell apoptosis, decreased cell proliferation and a better effect on the inhibition of angiogenesis compared with DTX alone.

Compared with the control group, tumor volumes were smaller and tumor wet weights were lighter in the USMB group. Immunohistochemical staining showed that mice treated with USMB demonstrated decreased cell proliferation and inhibited angiogenesis compared with the control group. The results of the present study indicated that USMB inhibits the growth of PC3 prostate cancer xenografts by inhibiting tumor cell proliferation and suppressing tumor angiogenesis, and this was consistent with the results of previous studies $(33,34)$.

In summary, the present study demonstrated marked enhancement of the antitumor effect of DTX through its combination with anti-vascular USMB. Compared with TACE, blood perfusion interruption mediated by USMB has several advantages. First, USMB can burst blood vessels and improve vascular permeability; drugs could go through the ruptured vessel wall and contact with tumor cells, thus increasing the concentration of the drugs around malignant cells. Second, USMB can increase the permeability of the cell walls by sonoporation $(28,35,36)$, which can facilitate the movement of drugs or genes into cells $(3,37)$, thereby increasing the concentration of the drugs in tumor cells. Third, USMB can induce diverse chemical and biological effects, including promoting cell apoptosis, and inhibiting cell proliferation and the neovasculature $(33,38)$. Fourth, USMB is simple to apply and is not susceptible to the complexities of the blood supply and the variations in blood vessel distribution.

There are certain limitations to the present study. First, the uptake of DTX by the tumor was not detected directly, so the dose of the drug delivered was not quantified. Second, the optimum drug dose and treatment intervals remain to be discussed. A dose-response curve should be performed in the future.

In conclusion, as a novel type of chemoembolization, USMB combined with DTX is more effective than USMB or DTX alone in inhibiting tumor growth, acting by the enhancement of apoptosis and the suppression of proliferation and angiogenesis. Based on these preclinical findings, it is suggested that USMB combined with chemotherapeutic drugs may be a promising alternative to TACE.

\section{Acknowledgements}

The authors would like to thank Runkun Pharmaceutical Corporation for providing the perfluoropropane-albumin microsphere injections. This study was supported by the
National Natural Science Foundation of China (grant nos. 81271597 and 81401421).

\section{References}

1. Siegel R, Ma J, Zou Z and Jemal A: Cancer statistics, 2014. CA Cancer J Clin 64: 9-29, 2014.

2. Goldberg AA, Titorenko VI, Beach A and Sanderson JT: Bile acids induce apoptosis selectively in androgen-dependent and -independent prostate cancer cells. PeerJ 1: e122, 2013.

3. Wang Y, Bai WK, Shen E and Hu B: Sonoporation by low-frequency and low-power ultrasound enhances chemotherapeutic efficacy in prostate cancer cells. Oncol Lett 6: 495-498, 2013.

4. Bahl A, Masson S, Birtle A, Chowdhury S and de Bono J: Second-line treatment options in metastatic castration-resistant prostate cancer: A comparison of key trials with recently approved agents. Cancer Treat Rev 40: 170-177, 2014.

5. Ganju A, Yallapu MM, Khan S, Behrman SW, Chauhan SC and Jaggi M: Nanoways to overcome docetaxel resistance in prostate cancer. Drug Resist Updat 17: 13-23, 2014.

6. Pienta KJ and Smith DC: Advances in prostate cancer chemotherapy: A new era begins. CA Cancer J Clin 55: 300-318; quiz 323-325, 2005

7. Sweeney CJ, Miller KD, Sissons SE, Nozaki S, Heilman DK, Shen J and Sledge GW Jr: The antiangiogenic property of docetaxel is synergistic with a recombinant humanized monoclonal antibody against vascular endothelial growth factor or 2-methoxyestradiol but antagonized by endothelial growth factors. Cancer Res 61: 3369-3372, 2001.

8. Wang Y, Hu B, Diao X and Zhang J: Antitumor effect of microbubbles enhanced by low frequency ultrasound cavitation on prostate carcinoma xenografts in nude mice. Exper Ther Med 3: 187-191, 2012.

9. Levenback BJ, Sehgal CM and Wood AK: Modeling of thermal effects in antivascular ultrasound therapy. J Acoust Soc Am 131: 540-549, 2012.

10. Hwang JH, Brayman AA, Reidy MA, Matula TJ, Kimmey MB and Crum LA: Vascular effects induced by combined $1-\mathrm{MHz}$ ultrasound and microbubble contrast agent treatments in vivo. Ultrasound Med Biol 31: 553-564, 2005.

11. Wood AK, Bunte RM, Price HE, Deitz MS, Tsai JH, Lee WM and Sehgal CM: The disruption of murine tumor neovasculature by low-intensity ultrasound-comparison between $1-$ and $3-\mathrm{MHz}$ sonication frequencies. Acad Radiol 15: 1133-1141, 2008.

12. Liu Z, Gao S, Zhao Y, Li P, Liu J, Li P, Tan K and Xie F: Disruption of tumor neovasculature by microbubble enhanced ultrasound: A potential new physical therapy of anti-angiogenesis. Ultrasound Med Biol 38: 253-261, 2012.

13. Goertz DE, Todorova M, Mortazavi O, Agache V, Chen B, Karshafian R and Hynynen K: Antitumor effects of combining docetaxel (taxotere) with the antivascular action of ultrasound stimulated microbubbles. PloS One 7: e52307, 2012.

14. Yang Y, Bai W, Chen Y, Lin Y and Hu B: Optimization of low-frequency low-intensity ultrasound-mediated microvessel disruption on prostate cancer xenografts in nude mice using an orthogonal experimental design. Oncol Lett 10: 2999-3007, 2015.

15. Coralic V and Colonius T: Shock-induced collapse of a bubble inside a deformable vessel. Eur J Mech B Fluids 40: 64-74, 2013.

16. Gao F, Hu Y and $\mathrm{Hu} \mathrm{H}$ : Asymmetrical oscillation of a bubble confined inside a micro pseudoelastic blood vessel and the corresponding vessel wall stresses. Int J Solids Struct 44: 7197-7212, 2007.

17. Gao F, Xiong $\mathrm{C}$ and Xiong Y: Constrained oscillation of a bubble subjected to shock wave in microvessel. Prog Nat Sci 19: 1109-1117, 2009.

18. Liu J, Xiao S, Wu S, Ou W, He J, Gao S and Liu Z: Disruption of splenic circulation using microbubble-enhanced ultrasound and prothrombin: A preliminary study. Ultrasound Med Biol 38: 1930-1937, 2012.

19. Moonen $C$ and Lentacker I: Ultrasound assisted drug delivery. Preface. Adv Drug Deliv Rev 72: 1-2, 2014.

20. Sorace AG, Warram JM, Umphrey $H$ and Hoyt $K$ : Microbubble-mediated ultrasonic techniques for improved chemotherapeutic delivery in cancer. J Drug Target 20: 43-54, 2012.

21. Nabili M, Shenoy A, Chawla S, Mahesh S, Liu J, Geist C and Zderic V: Ultrasound-enhanced ocular delivery of dexamethasone sodium phosphate: An in vivo study. J Ther Ultrasound 2: 6, 2014. 
22. Liu HL, Fan $\mathrm{CH}$, Ting $\mathrm{CY}$ and Yeh CK: Combining microbubbles and ultrasound for drug delivery to brain tumors: Current progress and overview. Theranostics 4: 432-444, 2014.

23. Kooiman K, Vos HJ, Versluis M and de Jong N: Acoustic behavior of microbubbles and implications for drug delivery. Adv Drug Deliv Rev 72: 28-48, 2014.

24. Chen H, Brayman AA, Bailey MR and Matula TJ: Blood vessel rupture by cavitation. Urol Res 38: 321-326, 2010.

25. Chen H, Kreider W, Brayman AA, Bailey MR and Matula TJ: Blood vessel deformations on microsecond time scales by ultrasonic cavitation. Phys Rev Lett 106: 034301, 2011.

26. Chen H, Brayman AA, Kreider W, Bailey MR and Matula TJ: Observations of translation and jetting of ultrasound-activated microbubbles in mesenteric microvessels. Ultrasound Med Biol 37: 2139-2148, 2011.

27. Dayton PA, Zhao S, Bloch SH, Schumann P, Penrose K, Matsunaga TO, Zutshi R, Doinikov A and Ferrara KW: Application of ultrasound to selectively localize nanodroplets for targeted imaging and therapy. Mol Imaging 5: 160-174, 2006.

28. Tzu-Yin W, Wilson KE, Machtaler S and Willmann JK: Ultrasound and microbubble guided drug delivery: Mechanistic understanding and clinical implications. Curr Pharm Biotechnol 14: 743-752, 2013

29. Karshafian R, Bevan PD, Williams R, Samac S and Burns PN: Sonoporation by ultrasound-activated microbubble contrast agents: Effect of acoustic exposure parameters on cell membrane permeability and cell viability. Ultrasound Med Biol 35: 847-860, 2009.

30. Jin LF, Li F, Wang HP, Wei F, Qin P and Du LF: Ultrasound targeted microbubble destruction stimulates cellular endocytosis in facilitation of adeno-associated virus delivery. Int $\mathbf{J}$ Mol Sci 14: 9737-9750, 2013.
31. Sheikov N, McDannold N, Sharma S and Hynynen K: Effect of focused ultrasound applied with an ultrasound contrast agent on the tight junctional integrity of the brain microvascular endothelium. Ultrasound Med Biol 34: 1093-1104, 2008.

32. Liu Y, Yi S, Zhang J, Fang Z, Zhou F, Jia W, Liu Z and Ye G: Effect of microbubble-enhanced ultrasound on prostate permeability: A potential therapeutic method for prostate disease. Urology 81: 921.e1-921.e7, 2013.

33. Eggen S, Fagerland SM, Mørch Ý, Hansen R, Søvik K, Berg S, Furu H, Bøhn AD, Lilledahl MB, Angelsen A, et al: Ultrasound-enhanced drug delivery in prostate cancer xenografts by nanoparticles stabilizing microbubbles. J Control Release 187: 39-49, 2014

34. Shen ZY, Shen E, Diao XH, Bai WK, Zeng MX, Luan YY, Nan SL, Lin YD, Wei C, Chen L, et al: Inhibitory effects of subcutaneous tumors in nude mice mediated by low-frequency ultrasound and microbubbles. Oncol Lett 7: 1385-1390, 2014.

35. Tomizawa M, Shinozaki F, Motoyoshi Y, Sugiyama T, Yamamoto $\mathrm{S}$ and Sueishi M: Sonoporation: Gene transfer using ultrasound. World J Methodol 3: 39-44, 2013.

36. Karshafian R, Samac S, Bevan PD and Burns PN: Microbubble mediated sonoporation of cells in suspension: Clonogenic viability and influence of molecular size on uptake. Ultrasonics 50: 691-697, 2010.

37. Yoon YI, Kwon YS, Cho HS, Heo SH, Park KS, Park SG, Lee SH Hwang SI, Kim YI, Jae HJ, et al: Ultrasound-mediated gene and drug delivery using a microbubble-liposome particle system. Theranostics 4: 1133-1144, 2014

38. Zhang B, Zhou H, Cheng Q, Lei L and Hu B: Low-frequency low energy ultrasound combined with microbubbles induces distinct apoptosis of A7r5 cells. Mol Med Rep 10: 3282-3288, 2014. 